\title{
Reduced long-term cost and increased patient satisfaction with rechargeable implantable pulse generators for deep brain stimulation
}

\author{
*Frederick L. Hitti, MD, PhD, Kerry A. Vaughan, MD, Ashwin G. Ramayya, MD, PhD, \\ Brendan J. McShane, BA, and Gordon H. Baltuch, MD, PhD \\ Department of Neurosurgery, Pennsylvania Hospital, University of Pennsylvania, Philadelphia, Pennsylvania
}

\begin{abstract}
OBJECTIVE Deep brain stimulation (DBS) has revolutionized the treatment of neurological disease, but its therapeutic efficacy is limited by the lifetime of the implantable pulse generator (IPG) batteries. At the end of the battery life, IPG replacement surgery is required. New IPGs with rechargeable batteries (RC-IPGs) have recently been introduced and allow for decreased reoperation rates for IPG replacements. The authors aimed to examine the merits and limitations of these devices.
\end{abstract}

METHODS The authors reviewed the medical records of patients who underwent DBS implantation at their institution. RC-IPGs were placed either during initial DBS implantation or during an IPG change. A cost analysis was performed that compared RC-IPGs with standard IPGs, and telephone patient surveys were conducted to assess patient satisfaction.

RESULTS The authors identified 206 consecutive patients from 2011 to 2016 who underwent RC-IPG placement (mean age 61 years; 67 women, 33\%). Parkinson's disease was the most common indication for DBS $(n=144,70 \%)$, followed by essential tremor $(n=41,20 \%)$, dystonia $(n=13,6 \%)$, depression $(n=5,2 \%)$, multiple sclerosis tremor $(n=2,1 \%)$, and epilepsy $(n=1,0.5 \%)$. DBS leads were typically placed bilaterally $(n=192,93 \%)$ and targeted the subthalamic nucleus $(n=136,66 \%)$, ventral intermediate nucleus of the thalamus $(n=43,21 \%)$, internal globus pallidus $(n=21,10 \%)$, ventral striatum $(n=5,2 \%)$, or anterior nucleus of the thalamus $(n=1,0.5 \%)$. RC-IPGs were inserted at initial DBS implantation in 123 patients $(60 \%)$, while 83 patients $(40 \%)$ were converted to RC-IPGs during an IPG replacement surgery. The authors found that RC-IPG implantation resulted in $\$ 60,900$ of cost savings over the course of 9 years. Furthermore, patient satisfaction was high with RC-IPG implantation. Overall, $87.3 \%$ of patients who responded to the survey were satisfied with their device, and only $6.7 \%$ found the rechargeable component difficult to use. In patients who were switched from a standard IPG to RC-IPG, the majority who responded (70.3\%) preferred the rechargeable IPG.

CONCLUSIONS RC-IPGs can provide DBS patients with long-term therapeutic benefit while minimizing the need for battery replacement surgery. The authors have implanted rechargeable stimulators in 206 patients undergoing DBS surgery, and here they demonstrate the cost-effectiveness and high patient satisfaction associated with this procedure. https://thejns.org/doi/abs/10.3171/2018.4.JNS172995

KEYWORDS deep brain stimulation; rechargeable; implantable pulse generator; battery; cost; patient satisfaction; functional neurosurgery

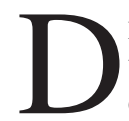
EEP brain stimulation (DBS) has revolutionized the treatment of several neurological disorders, including Parkinson's disease, essential tremor, and dystonia..$^{8,15,24,28,29}$ The introduction of DBS as a long-term treatment option has improved functional outcomes and quality of life for many patients., 40,16 Initial implementations of DBS relied on implantable pulse generators (IPGs) powered by nonrechargeable batteries. These devices have a limited lifespan and require an invasive procedure to remove and replace the IPG when the battery becomes

ABBREVIATIONS DBS = deep brain stimulation; IPG = implantable pulse generator; RC-IPG = rechargeable IPG .

SUBMITTED November 27, 2017. ACCEPTED April 12, 2018.

INCLUDE WHEN CITING Published online September 28, 2018; DOI: 10.3171/2018.4.JNS172995.

${ }^{*}$ F.L.H. and K.A.V. contributed equally to this work. 
depleted. Rechargeable technology has seen widespread implementation in consumer devices and has now been utilized in the medical device realm as well.

One strategy to limit number the number of IPG replacements would be to model battery lifespan and optimize DBS settings. Modeling, however, has not proven successful in predicting time to depletion and replacement of IPGs. ${ }^{21}$ Thus, patients are still required to present to their physician's office to check the voltage settings and lifespan of their DBS system. In addition to subjecting patients to the discomfort and pain of replacement IPG surgery, repeated replacements may also subject patients to an increased risk of complications. Some institutions have shown that as patients undergo more IPG replacement procedures, the risk of infection increases significantly. ${ }^{17,22}$ With the advent of rechargeable medical devices, these risks and limitations may be minimized. Furthermore, healthcare costs may be reduced by limiting IPG replacements.

In addition to IPGs for DBS, spinal cord stimulators also rely on batteries. Rechargeable batteries in the medical realm were first utilized by these devices. Both patients and physicians have reported high satisfaction with the rechargeable spinal cord stimulators, and patients have easily adapted to the requirement of recharging their IPG. ${ }^{16}$ With appropriate patient selection, the rechargeable spinal cord stimulator is becoming the system of choice given the high satisfaction. ${ }^{20}$ Following this example, rechargeable IPGs (RC-IPGs) were developed for DBS. The Activa $\mathrm{RC}$ neurostimulator (Medtronic) received FDA approval in 2009 and is currently available for clinical use in the US. The Brio neurostimulator (St. Jude Medical) and the Vercise stimulator (Boston Scientific) are 2 other RC-IPGs that have been developed, but they are currently not commercially available in the US. While not infinite, the lifespan of RC-IPGs is greater than the lifespan of standard IPGs, listed as 9 years in the US by manufacturers. This may confer the benefit of decreasing the number of IPG replacements and reducing cost.

After the introduction of the Medtronic RC-IPG, we adopted its use in our practice. We hypothesized that implantation of stimulators with rechargeable batteries would decrease cost while maintaining high patient satisfaction. Here, we detail our experience with this new device.

\section{Methods \\ Patient Selection}

We obtained approval from our institutional review board to retrospectively identify all consecutive patients at our institution who underwent DBS implantation with an RC-IPG during either a new implantation procedure or an IPG replacement procedure prior to May 2016. We use the Medtronic RC-IPG system for our DBS patients because of its availability in the US market. Any patient undergoing initial DBS implantation after April 2014 received a rechargeable neurostimulator unless either the patient or surgeon indicated a preference for a standard IPG. It was recommended that patients with severe disability (modified Rankin Scale score < 3) or end-stage movement disorders who lived in a facility or without adequate social/
TABLE 1. Patient characteristics

\begin{tabular}{lc}
\hline & Value \\
\hline Mean age at RC implant, yrs & $61 \pm 13^{*}$ \\
\hline Male & $139(67)$ \\
\hline Female & $67(33)$ \\
\hline Alive at time of survey & $202(98)$ \\
\hline Mean length of follow-up, yrs & $1.72 \pm 0.06 \dagger$ \\
\hline
\end{tabular}

Values are presented as the number of patients (\%) unless stated otherwise.

${ }^{*}$ Mean \pm SD.

$\dagger$ Mean \pm SEM

familial support to operate the rechargeable IPGs undergo placement of standard IPGs. All patients eligible for neurostimulator replacement surgery with the capability and reliability to operate the rechargeable IPG (or who had a caretaker to do so) were offered an RC-IPG.

\section{Clinical Data Collection}

Once the target patient population was identified, we collected relevant data from medical records, both paper and electronic (Epic, Epic Systems Corp.). Demographic information, indication for DBS treatment, and medical comorbidities were noted at the time of RC-IPG implantation. We also recorded the electrode implantation target and the number of subsequent replacement procedures after the index IPG implantation.

We also compiled surgical complications, including infection, significant discomfort from IPG requiring revision, or hematoma. Costs of representative procedures and devices were obtained from the billing department for the cost analysis. Phone surveys were conducted to determine DBS satisfaction. Likert-type response choices were used for the survey and were as follows: "completely agree," "mostly agree," "unsure," "mostly disagree," or "completely disagree." "Completely agree" and "mostly agree" were scored as affirmative responses. Patients' phone numbers were obtained from the medical record, and a minimum of 3 separate attempts were made to contact each patient.

\section{Statistical Analysis}

Statistical analysis was performed using Microsoft Excel and the GraphPad Prism software. Chi-square goodness-of-fit was used to determine significance of patient survey responses. Logistic regression analysis was performed using R. Results were considered significant if $p$ $<0.05$. Averages are presented as the mean \pm SEM unless otherwise specified.

\section{Results}

\section{Patient Population}

A total of 206 patients, the majority of whom were male $(n=139,67 \%)$, were included in the current study. The mean age at time of RC-IPG implantation was $61 \pm 13$ years $( \pm$ SD). By the endpoint of this study, 202 or $98 \%$ of the patients were alive. The length of follow-up was $1.72 \pm$ 0.06 years (Table 1). We also tracked our patients' comorbidities and found the most common to be hypertension, 


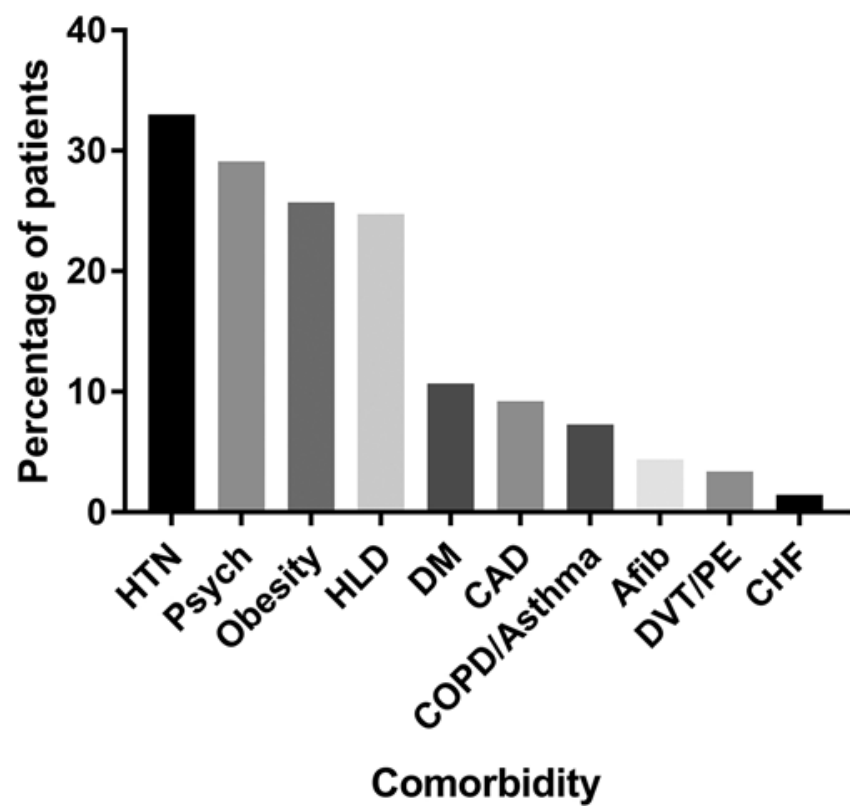

FIG. 1. Patient comorbidities as determined by chart review are listed in order of frequency. $\mathrm{HTN}=$ hypertension; Psych = psychiatric; $\mathrm{HLD}=$ hyperlipidemia; $\mathrm{DM}=$ diabetes mellitus; $\mathrm{CAD}=$ coronary artery disease; $\mathrm{COPD}=$ chronic obstructive pulmonary disease; Afib = atrial fibrillation; $\mathrm{DVT} / \mathrm{PE}=$ deep vein thrombosis or pulmonary embolus; $\mathrm{CHF}=$ congestive heart failure.

psychiatric disease (depression, bipolar, or anxiety), and obesity (Fig. 1). These diseases were present in 33\%, 29\%, and $26 \%$ of our patients, respectively.

\section{DBS Indications and IPG Types}

DBS may be used to treat a variety of disorders, and at our institution we routinely implant DBS systems in patients with Parkinson's disease, essential tremor, and dystonia (Table 2). The majority of DBS systems in the current study were implanted in patients with Parkinson's disease $(\mathrm{n}=144,70 \%)$, and the subthalamic nucleus was the most common target $136(66 \%)$. The internal globus pallidus as a target for Parkinson's disease was used in only 8 patients $(4 \%)$ in this cohort. Essential tremor was the next most common indication for treatment. We treated 41 patients (20\%) with this disorder. Electrodes were placed in the ventral intermediate nucleus of the thalamus in all essential tremor patients. While more rare, our patient population also included 2 patients treated for tremor due to multiple sclerosis by DBS implantation in the ventral intermediate nucleus. We have also been involved in several DBS clinical trials, including the Reclaim Deep Brain Stimulation Clinical Study for Treatment-Resistant Depression (clinicaltrials.gov, trial registration no. NCT00837486) and the SANTE (Stimulation of the Anterior Nucleus of the Thalamus for Epilepsy; clinicaltrials.gov, trial registration no. NCT00101933) trials. ${ }^{6,20}$ If the patients derived benefit from the DBS system in the open-label phase of the trial, they were offered IPG replacement surgery after battery depletion. Some of these study patients underwent implantation of an RC-IPG at time of IPG replacement and were included in this study. The majority of patients $(n=192$, 93\%) underwent bilateral DBS electrode placement.
TABLE 2. Indication for DBS and site of implantation

\begin{tabular}{lcl}
\hline \multicolumn{1}{c}{ Disorder } & No. of Patients (\%) & \multicolumn{1}{c}{ Site of Implant (\%) } \\
\hline Parkinson's disease & $144(70)$ & STN, 136 (66); GPi, 8 (4) \\
\hline Essential tremor & $41(20)$ & Vim, 41 (20) \\
\hline Dystonia & $13(6)$ & GPi, 13 (6\%) \\
\hline Depression & $5(2)$ & VC/VS, 5 (2) \\
\hline MS tremor & $2(1)$ & Vim, 2 (1) \\
\hline Epilepsy & $1(0.5)$ & ANT, 1 (0.5) \\
\hline
\end{tabular}

ANT = anterior nucleus of the thalamus; $\mathrm{GPi}=$ internal globus pallidus; $\mathrm{MS}=$ multiple sclerosis; STN = subthalamic nucleus; VC/VS = ventral capsule/ventral striatum; Vim = ventral intermediate nucleus.

In this study, we included patients who received an RCIPG at the time of initial DBS system implantation and patients who received an RC-IPG at time of IPG replacement surgery for battery depletion (Fig. 2). The majority of patients in this study $(123,60 \%)$ underwent implantation of an RC-IPG at time of initial system implantation. The remaining patients $(83,40 \%)$ underwent implantation of an RC-IPG at time of battery depletion. The latter cohort of patients initially had Kinetra $(38,18 \%)$, Activa PC (28, $14 \%)$, or Activa SC $(17,8 \%)$ stimulators before RC-IPG implantation (Fig. 2).

\section{Patient Satisfaction}

Patient telephone surveys were conducted to determine patient satisfaction with their DBS systems and, in particular, the rechargeable aspect of the system. There were 102 patients who completed the telephone survey $(50.5 \%$ response rate). The surveys were conducted an average of

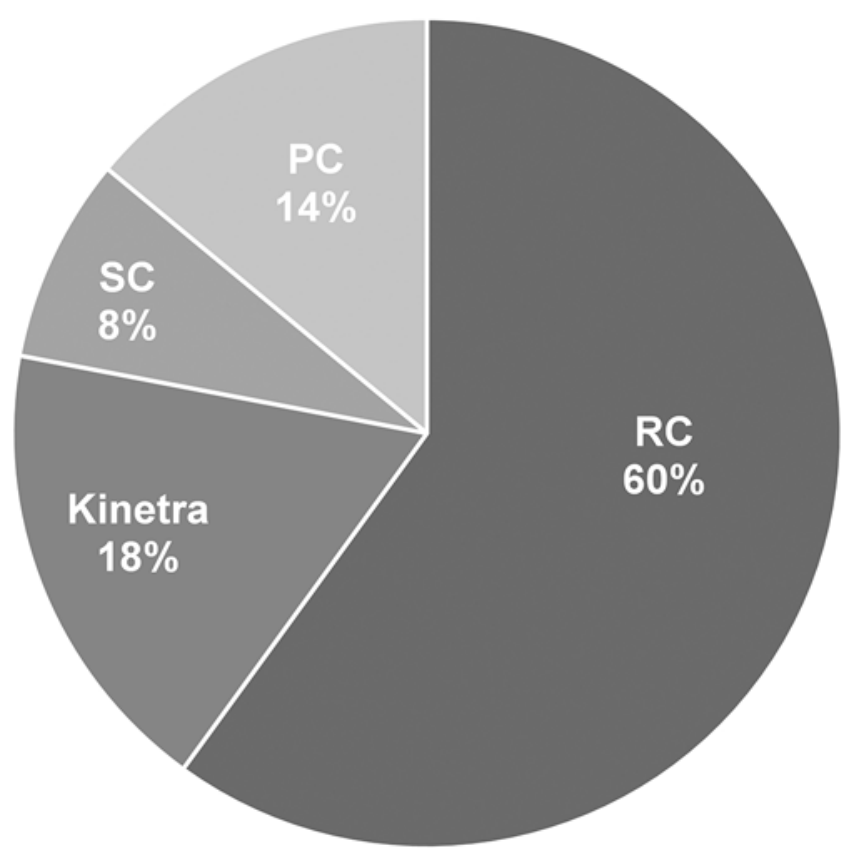

FIG. 2. Initial IPG. The majority of the patients in this study initially underwent implantation of an RC-IPG. In patients implanted with an RC-IPG at the time of battery depletion, the majority previously had Medtronic Kinetra or Activa PC stimulators. SC = Activa SC. 
TABLE 3. Patient satisfaction surveys in the 102 patients who responded to the survey

\begin{tabular}{lcc}
\hline \multicolumn{1}{c}{ Question } & $\begin{array}{c}\text { No. of Patients Replying } \\
\text { in Affirmative (\%) }\end{array}$ & $\begin{array}{c}p \\
\text { Value }\end{array}$ \\
\hline $\begin{array}{c}\text { The deep brain stimulator con- } \\
\text { trols my symptoms. }\end{array}$ & $78(76.5)$ & $<0.001$ \\
\hline $\begin{array}{l}\text { I would recommend this device } \\
\text { to someone else. }\end{array}$ & $93(91.2)$ & $<0.001$ \\
\hline I am happy with my DBS device. & $89(87.3)$ & $<0.001$ \\
\hline $\begin{array}{l}\text { I found the rechargeable battery } \\
\text { difficult to use. }\end{array}$ & $7(6.7)$ & $<0.001$ \\
\hline $\begin{array}{l}\text { I am happy that I have a re- } \\
\text { chargeable battery. }\end{array}$ & $81(79.4)$ & $<0.001$ \\
\hline $\begin{array}{l}\text { I prefer the rechargeable battery } \\
\text { compared to my old device. }\end{array}$ & $26(70.3)$ & 0.014 \\
\hline
\end{tabular}

${ }^{*}$ For patients converted to an RC-IPG ( $n=37$ survey respondents).

$27.6 \pm 0.8$ months after the initial RC-IPG implantation. We found that DBS implantation was associated with high satisfaction and subjective efficacy (Table 3). The majority of patients felt that the DBS system controlled their symptoms (76.5\%), would recommend the device to others (91.2\%), and were happy with their device (87.3\%). Importantly, very few patients found the rechargeable component of the system difficult to use (6.7\%), and the majority were happy that they had a rechargeable IPG (79.4\%). The surveys of patients who were switched from a standard IPG to an RC-IPG were especially informative. In this subset of patients $(\mathrm{n}=37)$, the majority $(70.3 \%)$ preferred the rechargeable IPG to the standard IPG (Table 3). The aforementioned distribution of survey responses was significantly different compared with an even distribution (half agree, half disagree) of responses. The $\mathrm{p}$ values were $<0.001,<0.001,<0.001,<0.001,<0.001$, and 0.014 , respectively.

While the majority of patients who switched from a standard IPG to an RC-IPG preferred the rechargeable IPG, a sizeable subset did not. The sex of patients in each subgroup was not significantly different $(\mathrm{p}=0.919,65.4 \%$ male in the group that preferred the RC-IPG vs $63.6 \%$ in the group that preferred the standard IPG). Diagnosis was also not significantly different between the 2 subgroups ( $\mathrm{p}$ $=0.125$, Wilcoxon rank-sum test). One of the most common reasons mentioned in office visits for standard IPG preference was the freedom from daily recharging.

\section{Cost Analysis}

Standard IPGs have a battery that is not rechargeable, and a replacement surgery is required at the end of battery life. The patient incurs the risks and costs of an additional procedure. Intuitively, fewer IPG replacements would result in cost savings. We examined the representative direct costs of implantation and replacement of a current-generation standard IPG (Activa PC) versus implantation of the RC-IPG (Activa RC). Both systems are capable of bilateral stimulation with a single IPG. The direct costs included the cost of hospitalization, procedure, and implanted devices.

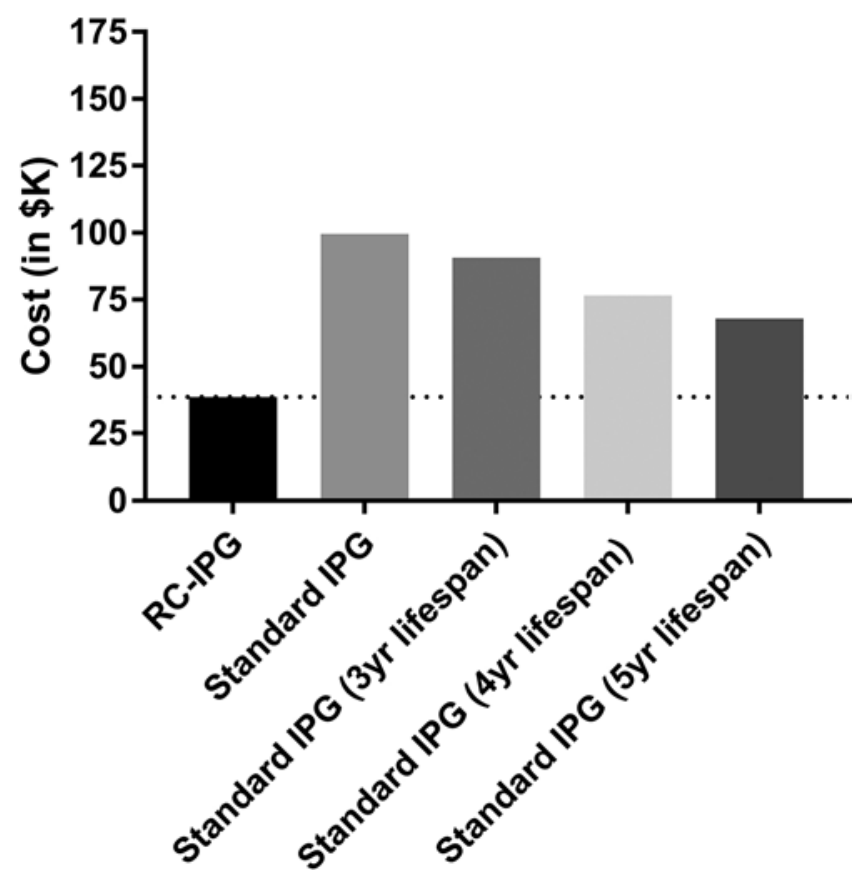

FIG. 3. IPG costs. RC-IPG implantation is associated with significant cost savings if long-term costs, including IPG replacements, are considered.

The representative direct cost of new DBS system implantation with an RC-IPG was $\$ 38,600$, while implantation with a standard IPG was slightly less expensive at $\$ 33,700$. The representative direct cost of standard IPG (Activa PC) replacement surgery was $\$ 19,000$. Regardless of procedure, device costs accounted for the vast majority of direct costs associated with DBS surgery (approximately $70 \%$ for new system implantation and approximately $90 \%$ for IPG replacement surgery).

As detailed above, 83 patients in the current study initially had a standard IPG and were implanted with an RCIPG during IPG replacement surgery for battery depletion. We performed a chart review to determine how many IPG replacements these patients had previously undergone to calculate an average IPG lifespan. Before RC-IPG implantation, these patients had their DBS systems in place for an average of $6.1 \pm 0.4$ years; they required an average of 2.7 \pm 0.2 IPG replacements during this time with an average IPG lifespan of $2.6 \pm 0.1$ years. The Activa RC is marketed as having a 9-year lifespan. Over the course of a 9-year period, the combined cost of standard IPG implantation followed by multiple IPG replacements is $\$ 99,500$. RC-IPG implantation, therefore, allows for a savings of $\$ 60,900$ over the course of 9 years (Fig. 3).

The cost calculations are very sensitive to the longevity of the IPGs. While we observed an IPG lifespan similar to that in our prior studies, other centers have demonstrated longer lifespans. ${ }^{11,25}$ For this reason, we also conducted the cost analysis for 3-, 4-, and 5-year potential standard IPG lifespans. The combined cost of standard IPG implantation followed by multiple IPG replacements is $\$ 90,700$ (3-year IPG lifespan), \$76,450 (4-year IPG lifespan), and $\$ 67,900$ (5-year IPG lifespan) (Fig. 3). The cost savings are $\$ 52,100, \$ 37,850$, and $\$ 29,300$, respectively. 
TABLE 4. Complications requiring reoperation

\begin{tabular}{cc}
\hline & No. of Patients (\%) \\
\hline Infection & \\
\hline Explant & $7(3)$ \\
\hline Washout & $8(4)$ \\
\hline Hematoma & $3(1)$ \\
\hline IPG site & $1(0.5)$ \\
\hline Intracerebral & $4(2)$ \\
\hline Hardware failure & $10(5)$ \\
\hline Repositioning of leads & $3(1)$ \\
\hline Repositioning of IPG
\end{tabular}

Some centers choose to implant 2 unilateral IPGs instead of 1 IPG capable of bilateral stimulation. The Medtronic Activa SC is one such IPG. The cost of new implantation of 2 Activa SC IPGs was similar to that of implantation of 1 Activa PC IPG (\$31,500 vs $\$ 33,700)$. Replacement of bilateral Activa SC IPGs totaled \$21,000. Hence, the combined cost of implantation with 2 Activa SC IPGs followed by multiple IPG replacements over a 9 -year interval is $\$ 104,200$. In this case, RC-IPG implantation results in a cost savings of $\$ 65,600$.

\section{Complications}

We tracked complications requiring reoperation in this cohort to determine if RC-IPG surgery was associated with an increased risk of complication compared with standard IPG surgery (Table 4). At our institution, we treat infections initially with IPG washout procedures, as most patients prefer to keep their DBS systems in place. Eight patients (4\%) required washout of a superficial infection. In patients in whom a washout fails or in patients who prefer to have the DBS system removed following infection, we offer explantation. Seven (3\%) of our patients underwent explantation. Two of these washouts and 1 of the system explantations were done in patients who underwent conversion of a standard IPG to an RC-IPG. To determine whether there was a significant increase in odds of infection for patients undergoing IPG replacement, we computed the odds ratio for infections in patients undergoing new IPG implantations and in patients undergoing IPG replacements. We found no significant difference in odds of infection between the 2 groups (OR 2.9, 95\% CI = $0.9-9.8, \mathrm{p}=0.11$ ).

We also asked if any of our patients' medical comorbidities increased the odds of developing an infection requiring washout or explantation. To investigate this, we conducted a logistic regression using the generalized linear model, followed by type II ANOVA. None of the medical comorbidities outlined in Fig. 1 significantly increased the likelihood of infection. The likelihood ratios and $p$ values, respectively, were as follows: atrial fibrillation, 1.33 and 0.25; CAD, 0.09 and 0.76; congestive heart failure, 1.77 and 0.18 ; chronic obstructive pulmonary disease, 0.20 and 0.59 ; diabetes mellitus, 0.44 and 0.51 ; hyperlipidemia, 0.26 and 0.61 ; deep vein thrombosis, 1.02 and 0.31 ; hypertension, 1.29 and 0.26; obesity, 0.00002 and > 0.99; and psychiatric, 0.51 and 0.47 .
A small percentage (1\%) of our patients underwent washout for IPG site hematoma, and 1 patient underwent evacuation of an intracerebral hematoma. In 4 (2\%) of our patients, high impedances were noted. Two of these patients were found to have fractured leads, and impedances improved after lead replacement. The other 2 patients underwent IPG replacement with improvement in impedances. Ten of our patients (5\%) underwent revision surgery for electrode or extension wire adjustment. The majority of these operations $(60 \%)$ were done for relief of patient discomfort associated with a sensation of neck pulling due to tight or scarred down extension wires. Four patients underwent reoperation for electrode repositioning in the brain. The RC-IPG must be placed in a particular orientation (with the printed text on the IPG facing anteriorly) so that it may be recharged externally. The RC-IPG must also be placed in a more superficial compartment (subcutaneous, not submuscular). Two patients underwent reoperation for placement of the IPG in the correct orientation. One patient was dissatisfied with the cosmetics of a subcutaneous IPG and opted for submuscular placement. The RC-IPG, however, could not be properly charged in this location and was changed to a standard IPG in accordance with the patient's wishes.

\section{Discussion}

DBS has revolutionized the treatment of many disorders, including Parkinson's disease, essential tremor, and dystonia, and has revitalized the field of functional neurosurgery. ${ }^{8,15,24,28,29}$ An unfortunate reality of DBS treatment is the need for IPG replacement, given the limited lifespan of the battery. In this study, we aimed to examine both the merits and shortcomings of the more recently developed RC-IPGs, which have a longer lifespan than standard IPGs.

We demonstrated that implantation of RC-IPGs is associated with a dramatic reduction in long-term cost and high patient satisfaction. We found that RC-IPG implantation resulted in a cost savings of $\$ 60,900$ over the course of 9 years. While the current marketed lifespan of the Medtronic Activa RC-IPG is 9 years, the actual lifespan may be even longer. In fact, the Boston Scientific RC-IPG (Vercise) that is available in Europe is marketed as having a 25-year lifespan, so the cost savings with this device would be even greater. While the manufacturers' advertised lifespans are encouraging, these numbers should be confirmed by examining the lifespan of implanted devices. The aforementioned cost reduction could be considered theoretical at this time, as actual RC-IPG lifespans may be shorter than their manufacturer-stated values. Shorter RC-IPG battery life would mitigate cost reduction. Other studies have also demonstrated reduced cost or number of IPG replacements associated with RC-IPG implantation in patients with Parkinson's disease, obsessive-compulsive disorder, and dystonia. ${ }^{3,9,18,19}$ The current study expands on these previous reports by examining a larger cohort of patients and confirming these findings.

We computed and compared the direct costs of RCIPG implantation to those of standard IPG implantation. Missing in these calculations are additional indirect and 
societal costs of standard IPG implantations. For example, there are many additional costs associated with repeated stimulator replacement surgeries. Caregivers and patients must take time off to present for clinic appointments and hospital visits for replacement surgery. The costs of preoperative planning for replacement surgeries should also be considered. In addition to cost savings, RC-IPG implantation is more environmentally friendly since standard IPG batteries are not generally recycled. Furthermore, due to the rechargeable nature of the device, clinicians may have fewer reservations regarding increasing the DBS stimulation strength for enhanced symptom control, as battery depletion becomes less of a concern.

RC-IPGs are more involved for patients than standard IPGs, as they require more diligent patient participation in treatment (vis-à-vis the charging process). Hence, the charging requirement may reduce patient satisfaction. However, we found that patient satisfaction with the RCIPG system remained high even after conversion from a standard IPG. Others have observed high patient satisfaction with RC-IPGs for the treatment of Parkinson's disease, essential tremor, and dystonia. ${ }^{13,14,23,27}$ Given the ubiquity of rechargeable consumer devices, the need to recharge likely has a minimal effect on patient satisfaction with RC-IPGs. It should also be noted that the time burden of recharging may increase over time as the rechargeable battery ages. The effect of this phenomenon on patient satisfaction merits further investigation as these relatively new devices age. Another potential cause of decreased patient satisfaction with RC-IPGs (when placed in patients who initially had standard IPGs) is the potential for decreased efficacy in symptom relief. A previous study noted that stimulation settings might have to be revised with the implantation of an RC-IPG. ${ }^{12}$

In addition to the benefits of cost savings and high patient satisfaction, the RC-IPG used in this study (Activa $\mathrm{RC}$ ) is much smaller than a standard IPG that is also capable of bilateral stimulation, such as the Activa PC. This cosmetic benefit, however, is tempered by the necessity of placing the IPG in a superficial subcutaneous pocket. As demonstrated above, the average lifespan of a nonrechargeable IPG was $2.6 \pm 0.1$ years in our cohort. While not included in the current cohort, DBS is also indicated in the treatment of children with dystonia. ${ }^{9}, 14,18$ RC-IPG implantation is especially beneficial in children as they would require many more IPG changes throughout their lifetimes and would benefit from the lower profile of the RC-IPG.

Previous studies have demonstrated an increase in infection rate during IPG replacement surgery compared with de novo IPG implantation. ${ }^{17,22}$ The increased morbidity of IPG replacement surgery would provide even more reason for the adoption of RC-IPGs. We did not observe this effect in our patient population. This increase may be due to a variety of reasons, such as surgical technique, patient population, or sampling error. Regardless, the cost reduction and high patient satisfaction are strong reasons to support RC-IPG implantation. RC-IPG implantation was also not associated with increased risk of complication compared with prior reports of DBS complication rates with standard IPGs. ${ }^{1,2,5,7,26,30}$ While some centers elect to implant 2 IPGs for bilateral stimulation, we typically use 1 IPG capable of bilateral stimulation. This holds true for both standard and rechargeable IPGs. Hence, in cases that required explantation, our experience with RC-IPGs was similar to our experience with standard IPGs.

The disadvantages of the RC-IPG stem from its need to be recharged. The wireless charging system requires the IPG and the charger to be relatively close. Hence, the IPG must be placed in a particular orientation and in a subcutaneous pocket. While 2 patients in the current study required reoperation for reorientation of the IPG, we have since implemented a surgical pause before IPG site closure to ensure that the IPG is in the correct orientation. Another consideration is that, at times, during replacement of a standard IPG with an RC-IPG, the existing chest pocket is larger than the new IPG. This has the potential to result in the IPG flipping to an incorrect orientation. For an RC-IPG, this would result in inability to charge and require reoperation. In these cases, we have stabilized the IPG in the correct orientation with suture. The more superficial placement of the RC-IPG necessary for charging also decreases cosmesis for some patients, exemplified by our patient who elected to undergo replacement of the RCIPG with a standard IPG because of cosmetic concerns. The RC-IPG system also requires more patient and family education than a standard IPG. Some of our patients and their families required multiple education sessions. In these cases, proper vendor support is critical. At our institution, the vendor representatives work closely with patients and their families on the day of surgery to provide a minimum of 2 education sessions. These education sessions are in addition to patient counseling offered in the outpatient setting. While a few patients required additional education sessions regarding the charging process, there were no overt IPG failures due to failure to recharge in a timely fashion. The efficacy of the system also relies on proper use and availability of the charging device, which may become misplaced or damaged. Again, proper vendor support is essential to ensure that patients are capable of securing another charger in a timely fashion. Finally, several of our patients noted defective antennas with early versions of the charging device. The charging devices have been recently upgraded to resolve this issue.

\section{Limitations}

Our study is limited by the retrospective nature of our methods. Longer follow-up in these RC-IPG patients will be crucial to determine whether they continue to be valuable alternatives to standard IPGs as this new generation of devices ages. A limitation of our patient satisfaction data is the incomplete response rate to our survey, as well as the lack of a fully validated scale to assess rechargeable battery satisfaction. We believe that the improvement in quality of life, the cost savings, and a complication rate that is similar to that of standard IPGs are clear advantages to the rechargeable devices, but these findings may benefit from corroboration by long-term studies. Our RC-IPG implantation protocol is also currently limited to patients who are able to comply with the charging requirements (or have families that can do so for them). Determining whether $\mathrm{RC}$ implantation would be feasible, safe, and beneficial in 
all types of patients undergoing DBS implantation merits further investigation.

\section{Conclusions}

RC-IPGs can provide DBS patients with long-term therapeutic benefit while minimizing the need for stimulator replacement surgery. Here, we have examined the merits and limitations of RC-IPGs in 206 patients undergoing DBS surgery. While some limitations remain, the cost-effectiveness and high patient satisfaction of these devices strongly argue for their continued use and further development.

\section{Acknowledgments}

We thank the members of the Neurosurgery Clinical Research Division (NCRD) for their assistance with IRB approval and data collection.

\section{References}

1. Bhatia S, Zhang K, Oh M, Angle C, Whiting D: Infections and hardware salvage after deep brain stimulation surgery: a single-center study and review of the literature. Stereotact Funct Neurosurg 88:147-155, 2010

2. Bjerknes S, Skogseid IM, Sæhle T, Dietrichs E, Toft M: Surgical site infections after deep brain stimulation surgery: frequency, characteristics and management in a 10 -year period. PLoS One 9:e105288, 2014

3. De Vloo P, Raymaekers S, van Kuyck K, Luyten L, Gabriëls L, Nuttin B: Rechargeable stimulators in deep brain stimulation for obsessive-compulsive disorder: a prospective interventional cohort study. Neuromodulation 21:203-210, 2018

4. Diamond A, Jankovic J: The effect of deep brain stimulation on quality of life in movement disorders. J Neurol Neurosurg Psychiatry 76:1188-1193, 2005

5. Doshi PK: Long-term surgical and hardware-related complications of deep brain stimulation. Stereotact Funct Neurosurg 89:89-95, 2011

6. Dougherty DD, Rezai AR, Carpenter LL, Howland RH, Bhati MT, O'Reardon JP, et al: A randomized sham-controlled trial of deep brain stimulation of the ventral capsule/ventral striatum for chronic treatment-resistant depression. Biol Psychiatry 78:240-248, 2015

7. Fernández-Pajarín G, Sesar A, Ares B, Relova JL, Arán E, Gelabert-González M, et al: Delayed complications of deep brain stimulation: 16-year experience in 249 patients. Acta Neurochir (Wien) 159:1713-1719, 2017

8. Follett KA, Weaver FM, Stern M, Hur K, Harris CL, Luo P, et al: Pallidal versus subthalamic deep-brain stimulation for Parkinson's disease. N Engl J Med 362:2077-2091, 2010

9. Gillies MJ, Joint C, Forrow B, Fletcher C, Green AL, Aziz TZ: Rechargeable vs. nonrechargeable internal pulse generators in the management of dystonia. Neuromodulation 16:226-229, 2013

10. Hälbig TD, Gruber D, Kopp UA, Schneider GH, Trottenberg T, Kupsch A: Pallidal stimulation in dystonia: effects on cognition, mood, and quality of life. J Neurol Neurosurg Psychiatry 76:1713-1716, 2005

11. Halpern CH, McGill KR, Baltuch GH, Jaggi JL: Longevity analysis of currently available deep brain stimulation devices. Stereotact Funct Neurosurg 89:1-5, 2011

12. Harries AM, Major S, Sandhu M, Honey CR: Rechargeable internal neural stimulators - is there a problem with efficacy? Neuromodulation 15:214-218, 2012

13. Jia F, Hao H, Meng F, Guo Y, Zhang S, Zhang J, et al: Patient perspectives on the efficacy of a new kind of rechargeable deep brain stimulators. Int J Neurosci 126:996-1001, 2016

14. Kaminska M, Lumsden DE, Ashkan K, Malik I, Selway R, Lin JP: Rechargeable deep brain stimulators in the management of paediatric dystonia: well tolerated with a low complication rate. Stereotact Funct Neurosurg 90:233-239, 2012

15. Kleiner-Fisman G, Liang GSL, Moberg PJ, Ruocco AC, Hurtig HI, Baltuch GH, et al: Subthalamic nucleus deep brain stimulation for severe idiopathic dystonia: impact on severity, neuropsychological status, and quality of life. J Neurosurg 107:29-36, 2007

16. Martínez-Martín P, Valldeoriola F, Tolosa E, Pilleri M, Molinuevo JL, Rumià J, et al: Bilateral subthalamic nucleus stimulation and quality of life in advanced Parkinson's disease. Mov Disord 17:372-377, 2002

17. Pepper J, Zrinzo L, Mirza B, Foltynie T, Limousin P, Hariz $\mathrm{M}$ : The risk of hardware infection in deep brain stimulation surgery is greater at impulse generator replacement than at the primary procedure. Stereotact Funct Neurosurg 91:5665, 2013

18. Perez J, Gonzalez V, Cif L, Cyprien F, Chan-Seng E, Coubes P: Rechargeable or nonrechargeable deep brain stimulation in dystonia: a cost analysis. Neuromodulation 20:243-247, 2017

19. Rizzi M, Messina G, Penner F, D'Ammando A, Muratorio F, Franzini A: Internal pulse generators in deep brain stimulation: rechargeable or not? World Neurosurg 84:1020-1029, 2015

20. Salanova V, Witt T, Worth R, Henry TR, Gross RE, Nazzaro $\mathrm{JM}$, et al: Long-term efficacy and safety of thalamic stimulation for drug-resistant partial epilepsy. Neurology 84:10171025,2015

21. Stewart CDM, Eljamel S: Prediction of implantable pulse generator longevity in deep brain stimulation: limitations and possible solutions in clinical practice. Stereotact Funct Neurosurg 89:299-304, 2011

22. Thrane JF, Sunde NA, Bergholt B, Rosendal F: Increasing infection rate in multiple implanted pulse generator changes in movement disorder patients treated with deep brain stimulation. Stereotact Funct Neurosurg 92:360-364, 2014

23. Timmermann L, Schüpbach M, Hertel F, Wolf E, Eleopra R, Franzini A, et al: A new rechargeable device for deep brain stimulation: a prospective patient satisfaction survey. Eur Neurol 69:193-199, 2013

24. Tröster AI, Fields JA, Pahwa R, Wilkinson SB, Strait-Tröster KA, Lyons K, et al: Neuropsychological and quality of life outcome after thalamic stimulation for essential tremor. Neurology 53:1774-1780, 1999

25. van Riesen C, Tsironis G, Gruber D, Klostermann F, Krause P, Schneider GH, et al: Disease-specific longevity of impulse generators in deep brain stimulation and review of the literature. J Neural Transm (Vienna) 123:621-630, 2016

26. Voges J, Waerzeggers Y, Maarouf M, Lehrke R, Koulousakis A, Lenartz D, et al: Deep-brain stimulation: long-term analysis of complications caused by hardware and surgery-experiences from a single centre. J Neurol Neurosurg Psychiatry 77:868-872, 2006

27. Waln O, Jimenez-Shahed J: Rechargeable deep brain stimulation implantable pulse generators in movement disorders: patient satisfaction and conversion parameters. Neuromodulation 17:425-430, 2014

28. Weaver FM, Follett K, Stern M, Hur K, Harris C, Marks WJ $\mathrm{Jr}$, et al: Bilateral deep brain stimulation vs best medical therapy for patients with advanced Parkinson disease: a randomized controlled trial. JAMA 301:63-73, 2009

29. Weaver FM, Follett KA, Stern M, Luo P, Harris CL, Hur $\mathrm{K}$, et al: Randomized trial of deep brain stimulation for Parkinson disease: thirty-six-month outcomes. Neurology 79:55-65, 2012 
30. Zhang J, Wang T, Zhang CC, Zeljic K, Zhan S, Sun BM, et al: The safety issues and hardware-related complications of deep brain stimulation therapy: a single-center retrospective analysis of 478 patients with Parkinson's disease. Clin Interv Aging 12:923-928, 2017

\section{Disclosures}

The authors report no conflict of interest concerning the materials or methods used in this study or the findings specified in this paper.

\section{Author Contributions}

Conception and design: Hitti, Vaughan, Ramayya, Baltuch. Acqui- sition of data: McShane. Analysis and interpretation of data: Hitti, Vaughan, Baltuch. Drafting the article: Hitti, Vaughan, Ramayya. Critically revising the article: all authors. Reviewed submitted version of manuscript: Hitti, Vaughan, Ramayya, Baltuch. Approved the final version of the manuscript on behalf of all authors: Hitti. Statistical analysis: Hitti. Administrative/technical/ material support: Baltuch. Study supervision: Baltuch.

\section{Correspondence}

Frederick Hitti: University of Pennsylvania, Philadelphia, PA. frederick.hitti@uphs.upenn.edu. 